\title{
Geometrical Optics of Beams with Vortices: Berry Phase and Orbital Angular Momentum Hall Effect
}

\author{
Konstantin Yu. Bliokh ${ }^{1,2,3}$ \\ ${ }^{1}$ Institute of Radio Astronomy, Kharkov, 61002, Ukraine \\ ${ }^{2}$ A.Ya. Usikov Institute of Radiophysics and Electronics, Kharkov, 61085, Ukraine \\ ${ }^{3}$ Department of Physics, Bar-Ilan University, Ramat Gan, 52900, Israel
}

\begin{abstract}
We consider propagation of a paraxial beam carrying the spin angular momentum (polarization) and intrinsic orbital angular momentum (IOAM) in a smoothly inhomogeneous isotropic medium. It is shown that the presence of IOAM can dramatically enhance and rearrange the topological phenomena that previously were considered solely in connection to the polarization of transverse waves. In particular, the appearance of a new-type Berry phase that describes the parallel transport of the beam structure along a curved ray is predicted. We derive the ray equations demonstrating the splitting of beams with different values of IOAM. This is the orbital angular momentum Hall effect, which resembles Magnus effect for optical vortices. Unlike the recently discovered spin Hall effect of photons, it can be much larger in magnitude and is inherent to waves of any nature. Experimental means to detect the phenomena is discussed.
\end{abstract}

PACS numbers: 41.20.Jb, 03.65.Vf, 41.85.-p, 42.15.-i

Introduction. The total angular momentum (TAM) of an electromagnetic wave packet (or a beam) can be presented in the form of three summands: TAM $=\mathrm{EOAM}+\mathrm{IOAM}+\mathrm{SAM}$. Here IOAM stands for the intrinsic orbital angular momentum of the packet, i.e. the orbital angular momentum relative to the center of gravity of the packet, EOAM is the extrinsic orbital angular momentum related to the motion of the center of gravity, and SAM is the spin angular momentum determined by the polarization of the wave packet. While EOAM and SAM are well known and studied during many decades, the beams carrying IOAM evoked intensive theoretical and experimental investigations only in last 14 years [1]. This fact is connected to the contemporary laboratory resources for the generation and transformation of light beams as well as single photons with non-zero values of IOAM. The most popular beams with IOAM are the Laguerre-Gaussian beams which contain the optical vortices along their axes and form a complete, orthogonal, basic set from which an arbitrary field distribution can de described. The states of light with well-defined IOAM offer now exciting possibilities for the optical manipulation with matter, study of the entanglement of photons, and a lot of problems of the classical electrodynamics [1].

Simultaneously, in the past decade, the topological phenomena related to the spin of particles caused a great activity in the various areas of physics: condensed matter, high energy physics, optics. It is caused by both fundamental character of the problems and promising applications in new areas of nanotechnologies, such as spintronics, photonics, etc. Nontrivial evolution of the spin states in the semiclassical approximation can be treated by means of the spin-orbit interaction, which is the coupling between SAM and EOAM. Spin-orbit interaction leads to the mutual influence of the polarization and the trajectory of the particle's motion, and produces two re- ciprocal topological phenomena. These are the geometrical Berry phase 2, 33 and recently discovered topological spin transport or intrinsic spin Hall effect [4]. In the geometrical optics, the Berry phase provides for the parallel transport of the polarization vector along the ray [3], whereas the spin Hall effect manifests itself as the transverse deflection of polarized beams when propagating in an inhomogeneous medium. The latter phenomenon includes the polarization-dependent transverse Fedorov-Imbert shift in the reflection or refraction of the beam on a sharp boundary [5, 6, 8, 10], and the splitting of rays of different polarizations in a smoothly inhomogeneous medium [ $6,7,7,8,[9]$. The common roots of these effects and their connection to the spin-orbit interaction and TAM conservation are indicated in the papers [6, [8, 10].

All mentioned phenomena are related to the interaction between the polarization of light (SAM of the field) and its extrinsic orbital features, EOAM. At the same time, the presence of the IOAM in the beam apparently may lead to analogous effects. Then large values of IOAM can give a great advantage compared to small polarization phenomena constrained by unit value of SAM per photon $(\hbar=1)$. The transverse Fedorov-Imbert shift related to IOAM has been recently described and measured 11]. In the present paper, similarly to the developed earlier geometrical optics with the spin-orbit interaction [6, 7, 8, 9], we evolve the geometrical optics with the orbit-orbit interaction, i.e., an interaction between IOAM and EOAM of the beam propagating along a curved ray.

Berry connection and curvature. We consider a monochromatic paraxial electromagnetic beam with definite values of SAM and IOAM, which propagates in a smoothly inhomogeneous isotropic medium. Locally, the beam's electric field (without $e^{-i \omega t}$ factor) can be repre- 
sented as in homogeneous medium:

$$
\mathbf{E}^{p, l, \sigma}=\mathbf{e}^{\sigma} F^{p,|l|}(\rho) \exp \left[i l \varphi+i \int k d s\right] .
$$

Here $\mathbf{k}(s)$ is the central wave vector directed along the beam $s$ axis; $(\rho, \varphi, s)$ are the local cylindrical coordinates following the central ray whose trajectory is described by the geometrical optics ray equations; $\mathbf{e}^{\sigma}$ is the unit vector of the polarization of wave with the helicity $\sigma= \pm 1$ which is the value of $s$-directed SAM per photon $\left(\mathbf{e}^{ \pm}\right.$ correspond to waves of right and left circular polarizations, so that $\mathbf{e}^{ \pm *}=\mathbf{e}^{\mp}$ and $\left.\mathbf{e}^{\sigma \dagger} \mathbf{e}^{\sigma^{\prime}}=\delta^{\sigma \sigma^{\prime}}\right) ; F^{p,|l|}$ is the radial function with quantum number $p=0,1,2, \ldots$; finally, $l=0, \pm 1, \ldots, \pm p$ is the azimuthal quantum number which is the value of $s$-directed IOAM per photon. The expression (1) is given in a diffractionless approximation and does not account for variations of the beam's envelope, phase front, and the Guoy phase; as it is known, the diffraction phenomena do not contribute to the geometrical optics characteristics of the wave packet evolution. In the paraxial approximation one can consider the longintudinal wave vector $k$ as independent of the transverse structure of the wave field and the polarization vector $\mathbf{e}^{\sigma}$ as common for the whole beam. This is the paraxial approximation that enables one to consider the states of field with simultaneously well-defined values of the helicity (SAM) and IOAM [1]. The axis of the beam (11) contains an optical dislocation (the phase singularity): the optical vortex of the strength $l$. The scalar product for beams (1) is defined as for vectors in Hilbert space, trough integration over the beam crosssection with $s$ replacing 'time' 1]; we assume that beams (11) form an orthonormal basis: $\left(\mathbf{E}^{p, l, \sigma}, \mathbf{E}^{p^{\prime}, l^{\prime}, \sigma^{\prime}}\right) \equiv$ $\iint \mathbf{E}^{p, l, \sigma \dagger} \mathbf{E}^{p^{\prime}, l^{\prime}, \sigma^{\prime}} \rho d \rho d \varphi=\delta^{p p^{\prime}} \delta^{l l^{\prime}} \delta^{\sigma \sigma^{\prime}}$.

The variations in the ray direction, $\mathbf{k}$, give rise to a nontrivial parallel transport law. It is determined by the Berry connection (gauge potential) defined as

$$
\mathcal{A}^{\sigma \sigma^{\prime} l l^{\prime}}(\mathbf{k})=i\left(\mathbf{E}^{p, l, \sigma}, \frac{\partial}{\partial \mathbf{k}} \mathbf{E}^{p, l^{\prime}, \sigma^{\prime}}\right)
$$

(the radial index contributes to the Berry connection in a trivial way and is not considered). Equation (2) with the field (11) yields $\mathcal{A}^{\sigma \sigma^{\prime} l l^{\prime}}=\delta^{l l^{\prime}} \mathcal{A}^{\sigma \sigma^{\prime}}+\delta^{\sigma \sigma^{\prime}} \mathcal{A}^{l l^{\prime}}$. Here $\mathcal{A}^{\sigma \sigma^{\prime}}=i \mathbf{e}^{\sigma \dagger} \frac{\partial}{\partial \mathbf{k}} \mathbf{e}^{\sigma^{\prime}}$ is the known spin-related Berry connection for plane waves that accounts for the parallel transport of the polarization vector in the $\mathbf{k}$-space [3], whereas $\mathcal{A}^{l l^{\prime}}=i \int_{0}^{2 \pi} e^{-i l \varphi} \frac{\partial}{\partial \mathbf{k}} e^{i l^{\prime} \varphi} d \varphi$ is a new term connected to the IOAM. The spin term is a diagonal (Abelian) gauge potential: $\mathcal{A}^{\sigma \sigma^{\prime}}=\delta^{\sigma \sigma^{\prime}} \mathcal{A}^{\sigma}=\delta^{\sigma \sigma^{\prime}} \sigma \mathcal{A}$, which gives rise to the gauge field (Berry curvature) of the magnetic monopole type: $\mathcal{F}^{\sigma \sigma^{\prime}}=\delta^{\sigma \sigma^{\prime}} \mathcal{F}^{\sigma}=\delta^{\sigma \sigma^{\prime}} \sigma \mathcal{F}$, $\mathcal{F}=\frac{\partial}{\partial \mathbf{k}} \times \mathcal{A}=-\frac{\mathbf{k}}{k^{3}}[3,7,8]$. By evaluating the orbit term, we derive $\mathcal{A}^{l l^{\prime}}=\delta^{l l^{\prime}} l \mathcal{A}$. Finally, the total Berry connection and curvature $\mathcal{F}^{\sigma \sigma^{\prime} l l^{\prime}}=\frac{\partial}{\partial \mathbf{k}} \times \mathcal{A}^{\sigma \sigma^{\prime} l l^{\prime}}$ read:

$\mathcal{A}^{\sigma \sigma^{\prime} l l^{\prime}}=\delta^{\sigma \sigma^{\prime}} \delta^{l l^{\prime}}(\sigma+l) \mathcal{A}, \mathcal{F}^{\sigma \sigma^{\prime} l l^{\prime}}=\delta^{\sigma \sigma^{\prime}} \delta^{l l^{\prime}}(\sigma+l) \mathcal{F}$.

Thus, the Berry connection for beams (11) is also a diagonal Abelian potential represented by a tensor of higher rank. Propagation in a smoothly inhomogeneous medium keeps the beam's helicity, $\sigma$, and IOAM, $l$, conserved in the geometrical optics approximation. Hence, the Berry connection and curvature for a given beam (11) equal $\mathcal{A}^{\sigma l}=(\sigma+l) \mathcal{A}$ and $\mathcal{F}^{\sigma l}=(\sigma+l) \mathcal{F}=-(\sigma+l) \mathbf{k} / k^{3}$. Thus, topologically, a beam with IOAM behaves similarly to a polarized beam containing only SAM; however, the magnitude of the phenomena changes: instead of SAM, $\sigma$, now they are proportional to the total intrinsic angular momentum of the beam: SAM+IOAM, $\sigma+l$. In contrast to situations considered previously, the nontrivial topological features can be manifested by beams without SAM, $\sigma=0$, in particular by beams of longitudinal (sound) waves with $l \neq 0$.

Geometrical phase and parallel transport of the beam structure. We proceed to describe the basic phenomena arising from the Berry connection (3). On the evolution, the beam acquires an additional phase, namely, the Berry geometrical phase that equals

$$
\Theta_{B}^{\sigma l}=\int_{C} \mathcal{A}^{\sigma l} d \mathbf{k}=(\sigma+l) \int_{C} \mathcal{A} d \mathbf{k}=(\sigma+l) \Theta_{B 0},
$$

where $C$ is the contour of the ray evolution in the $\mathbf{k}$ space, and $\Theta_{B 0}$ is the Berry phase accumulated in the beam with $\sigma=1$ and $l=0$. For a cyclic evolution in the k-space, when $C$ is a loop, the Berry phase can be represented as a surface integral of the Berry curvature: $\Theta_{B}^{\sigma l}=(\sigma+l) \oint_{S} \mathcal{F} d \mathbf{k}=-(\sigma+l) \Omega$, where $C=\partial S$, and $\Omega$ is the solid angle at which contour $C$ is seen from the origin $\mathbf{k}=0$. With the Berry phase (4) taken into account, the field (1) of the beam propagating along a curved ray should be rewritten as

$$
\mathbf{E}^{p, l, \sigma}=\mathbf{e}^{\sigma} e^{i \sigma \Theta_{B 0}} F^{p,|l|} \exp \left[i l\left(\varphi+\Theta_{B 0}\right)+i \int k d s\right] .
$$

This equation is one of the central results of the paper. There factor $e^{i \sigma \Theta_{B 0}}$ is the known Berry phase associated with the parallel transport of the polarization vector 3], while the new-type 'orbital' Berry phase, incorporated into the azimuthal distribution, shows that on the evolution, the transverse distribution of the field rotates at the angle $-\Theta_{B 0}$, which corresponds to the parallel transport of the beam structure along the ray. It follows from Eq. (5) that an arbitrary electromagnetic beam representable as a superposition of beams (1), e.g. a HermiteGaussian beam, will also experience a rotation at the angle $-\Theta_{B 0}$ in accordance with the parallel transport law, Fig. 1 [12]. The rotation of the transverse intensity 


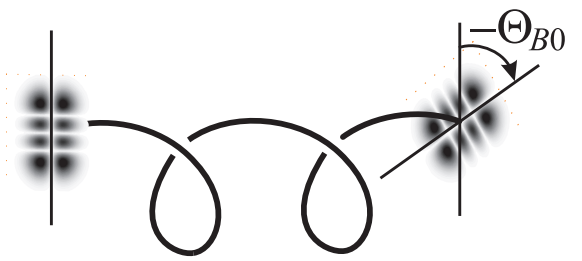

FIG. 1: The parallel transport of the cross-structure of a beam ( $\mathrm{HG}_{31}$-mode here) propagating along a helical trajectory.

distribution in the beam can be observed in propagation of a Hermite-Gaussian beam along a helical ray in an axially symmetric medium or in a circular multimode optical fiber stranded in a helix. In the latter case, the eigen modes of the fiber have the form of Eq. (11) 13, 14] and, hence, the speckle pattern at the output of the fiber will rotate depending on its torsion [15]. In the fiber, the eigen modes (11) experience no diffraction, which will be helpful when detecting the effect 12].

Ray equations: spin and orbital Hall effects. The ray equations, which describe the motion of the center of the beam (5) in a smoothly inhomogeneous medium, are Hamiltonian semiclassical equations of motion. They have been derived a number of times for various particles in the presence of the Berry curvature in the $\mathbf{k}$-space [2, 4, 6, 6, 8, 9]. Completely similarly to the case of the spin-related Berry curvature [6, 7, [8, 9], we obtain:

$\dot{\mathbf{k}}=k \nabla \ln n, \dot{\mathbf{r}}=\frac{\mathbf{k}}{k}+\mathcal{F}^{\sigma l} \times \dot{\mathbf{k}}=\frac{\mathbf{k}}{k}-(\sigma+l)\left(\frac{\mathbf{k}}{k^{3}} \times \dot{\mathbf{k}}\right)$,

where dot denotes the derivative with respect to $s$, and $n(\mathbf{r})$ is the refractive index of the medium. Equations (6) are the second central results of the paper. They differ from the 'traditional' ray equations of the geometrical optic by the term proportional to the Berry curvature in the right-hand side of the second equation. This term is referred to as "anomalous velocity" (since it contributes to $\dot{\mathbf{r}}$ ) [4]; it gives rise to the ray deflection, $\delta \mathbf{r}^{\sigma l}$, which crucially depends on the polarization and IOAM of the beam, Fig. 2. When the anomalous velocity does not contribute to $\mathbf{k}$, the deflection, like the Berry phase, can be represented in the form of a contour integral in the $\mathbf{k}$ space: $\delta \mathbf{r}^{\sigma l}=\int_{C} \mathcal{F}^{\sigma l} \times d \mathbf{k}=-(\sigma+l) \int_{C} \frac{\mathbf{k} \times d \mathbf{k}}{k^{3}}$. Here contour $C$ is determined by the rays of zero approximation, i.e. by Eqs. (6) with $\dot{\mathbf{r}}=\mathbf{k} / k$. The deflection is small in magnitude (it is proportional to the wavelength) but can grow unlimitedly with the ray length $s$; its maximum value can be estimated as $\left|\delta \mathbf{r}^{\sigma l}\right|_{\max } \sim|\sigma+l| s / k L$, where $L$ is the characteristic scale of the medium inhomogeneity. Since the anomalous velocity is directed orthogonally to both the main beam propagation direction, $\mathbf{k}$, and the applied 'external force' $\mathbf{k} \propto \nabla n$ (Fig. 2 ), the phenomenon can be treated as intrinsic Hall effect of photons, which is of a universal nature for var- ious particles [4]. If one separates the spin and orbital parts in an obvious way, $\delta \mathbf{r}^{\sigma l}=\delta \mathbf{r}^{\sigma}+\delta \mathbf{r}^{l}$, the previously known SAM-related deflection $\delta \mathbf{r}^{\sigma}$ can be associated with the spin Hall effect of photons [6, 7, 8, 9], whereas the IOAM-related deflection $\delta \mathbf{r}^{l}$, introduced in the present paper, represents a novel effect - intrinsic orbital angular momentum Hall effect. Indeed, the splitting of beams (5) with different (opposite) values of $l$ implies the appearance of the transverse current of IOAM (Fig. 2) [16]. Various examples of media, where the spin Hall effect gives rise to noticeable transport of rays, can be found in papers $[\underline{6}, \mathbf{7},[8]$; due to mathematical similarity of ray equations, the IOAM Hall effect in those media can be considered analogously. We emphasize that the presence of IOAM dramatically enhances the topological transport described by Eqs. (6), since the value of $l$ can reach tens and even hundreds. The IOAM Hall effect can be much more efficient as compared with the spin Hall effect and can be observed independently on the wave polarization.

Since the center of the beam (5) is a vortex core, Eqs. (6) can be regarded as the equations for the field dislocation line in an inhomogeneous medium. Then, deflection $\delta \mathbf{r}^{\sigma l}$ strongly resembles the effect of Magnus force acting on a vortex in superconductors, where it also directly related to the Berry phase but in $\mathbf{r}$ - rather than $\mathbf{k}$-space 17] (see also 14]). Association with the vortex line gives a good opportunity for measuring $\delta \mathbf{r}^{\sigma l}$, since the singularity can be observed with a great accuracy.

TAM conservation and Fedorov-Imbert shift. TAM of the paraxial beam (11) (including EOAM) can be represented in the form $\mathbf{J}=\mathbf{r} \times \mathbf{k}+(\sigma+l) \mathbf{k} / k$. It can be easily shown (see $[4,8]$ ), that it is the Berry curvature term in the equations of motion (6) that guarantees conservation of $J_{z}$ in an axially symmetrical with respect to $z$ axis medium, as well as conservation of $\mathbf{J}$ in a medium spherically symmetric with respect to the origin. The conservation of TAM of the beam reveals the common nature of the transport under consideration and the transverse Fedorov-Imbert shift at a sharp interface between two media. The latter problem has been analyzed rigorously for paraxial beams with IOAM in [11]. When a Laguerre-Gaussian beam experiences scattering at the interface with a low contrast $\delta n \ll 1$, the reflected beam can be neglected, and the transverse shift of the refracted beam approximately equals [1] $\delta x \approx \frac{(\sigma+l)}{k} \frac{\delta n}{n} \tan \vartheta$. Here $\vartheta$ is the angle of incidence, and $x$ axis points orthogonally to both $\mathbf{k}$ and the normal to the interface $(\nabla n)$. Precisely the above shift provides for conservation of the normal component of $\mathbf{J}[\underline{5}$, [8, 10]. In the differential limit, $\delta n \rightarrow d n, \delta x \rightarrow d x$, it leads to the ray equations (6) in a smoothly inhomogeneous medium, see [6].

Evolution of beams superposition. Suppose now that the field presents a superposition of beams (11) with different quantum numbers: $\mathbf{E}=A \sum_{p, l, \sigma} a^{p l \sigma} \mathbf{E}^{p, l, \sigma}$, where the common amplitude $A$ is chosen in such a way that 
$\sum_{p, l, \sigma}\left|a^{p l \sigma}\right|^{2}=1$. A set of amplitudes $a^{p l \sigma}$ represents the unit state vector in the basis of beams (11) 1]. Then, one can determine the expected value of any quantity for the superposition state. Since the Berry gauge field is Abelian, the transition to the expected values is realized in a straightforward way. The ray equations for expected values, $\overline{\mathbf{r}}$ and $\overline{\mathbf{k}}$, (which are the coordinates of the center of gravity of the total beam in the phase space), takes the form similar to Eqs. (6) where one component of the Berry curvature $\mathcal{F}^{\sigma l}$ is replaced with the convolution $\overline{\mathcal{F}}=\sum_{p, l, l^{\prime}, \sigma, \sigma^{\prime}} a^{p l \sigma *} \mathcal{F}^{\sigma \sigma^{\prime} l l^{\prime}} a^{p l^{\prime} \sigma^{\prime}}=\sum_{p, l, \sigma}\left|a^{p l \sigma}\right|^{2} \mathcal{F}^{\sigma l}$, and quantum numbers $\sigma$ and $l$ are replaced by 'classical' quantities $\bar{\sigma}=\sum_{p, l, \sigma}\left|a^{p l \sigma}\right|^{2} \sigma$ and $\bar{l}=\sum_{p, l, \sigma}\left|a^{p l \sigma}\right|^{2} l$. It is worth noticing that though the evolution of the beam's center of gravity is described by the expected values, in actual fact the total beam splits into the partial beams propagating along slightly shifted trajectories (6). This can be detected, as in the Hall effects, through accumulation of the photon states with opposite signs of $\sigma$ or $l$ at the opposite sides of the beam, see 10, 18], or through the splitting of the singularity lines of vortices with different $l$. In this way, a Hermite-Gaussian beam propagating in an inhomogeneous medium will split into composing Laguerre-Gaussian beams (1) with opposite values of $l$ (the birth of vortex pairs will accompany this process). Like the circularly rather than linearly polarized plane waves are independent modes in an inhomogeneous medium [7, 10, 18], it is the Laguerre-Gaussian beams that constitute the independent localized modes. This follows from the diagonality of the Berry connection (3) in the basis of beams (11).

When a superposition of beams (11) is considered, their Berry phases introduced explicitly in Eq. (5) can be taken into account by means of the equation of evolution of the state vector $a^{p l \sigma}$. Analogously to the equation of motion for the state vector with one quantum number $\sigma$ [8], in general case we have:

$$
\dot{a}^{p l \sigma}=i \sum_{l^{\prime}, \sigma^{\prime}} \mathcal{A}^{l l^{\prime} \sigma \sigma^{\prime}} \dot{\mathbf{k}} a^{p l^{\prime} \sigma^{\prime}}=i \mathcal{A}^{l \sigma} \dot{\mathbf{k}} a^{p l \sigma} .
$$

Integration of Eq. (77) gives the Berry phases acquired by the partial beams, $a^{p l \sigma}=a_{0}^{p l \sigma} \exp \left[i(\sigma+l) \Theta_{B 0}\right]$, and the parallel transport of the beam's field.

Conclusion. We have considered propagation of a paraxial beam carrying intrinsic spin and orbital angular momenta in a smoothly inhomogeneous medium in the geometrical optics approximation. It is shown that expressions for the Berry connection and curvature, as well as the equations of motion, are similar to situation when only SAM is presented, with a substitution $\sigma \rightarrow \sigma+l$. However, there are some sharp distinctions. First, the additional Berry phase acquired during the beam evolution provides for the parallel transport of the intensity cross

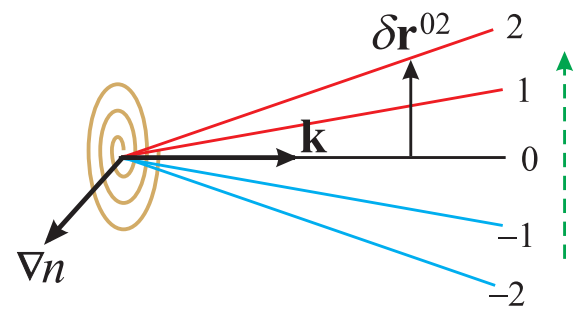

FIG. 2: (color online). Transverse transport of rays (vortex cores) in an inhomogeneous medium. The rays are marked with values of $\sigma+l$. The dashed arrow points the direction of the intrinsic angular momentum current.

distribution in the beam along the ray. The effect can be detected when a Hermite-Gaussian beam propagates of along a helical trajectory or through the measurement of rotation of the speckle-pattern when a circular multimode fiber is twisted. Second, the ray equations describing deflections of beams with different values of $\sigma+l$, predicts thereby the intrinsic orbital angular momentum Hall effect for photons, which resembles Magnus effect for optical vortices. The effect has significant advantages compared to the spin Hall effect of photons: (i) it can be dozen of times larger in magnitude with the respective values of $l$; (ii) it is independent of the polarization and takes place for waves of any nature. The effect can be measured through the deflection or splitting of singularity lines for vortices with different $l$.

[1] Optical Angular Momentum, edited by L. Allen, S.M. Barnett, and M.J. Padgett (Taylor and Francis, 2003).

[2] M.V. Berry, Proc. R. Soc. Lond. A 392, 45 (1984); A. Bohm et al., Geometrical Phase in Quantum Systems (Springer Verlag, 2003).

[3] S.I. Vinnitski et al., Usp. Fiz. Nauk 160(6), 1 (1990) [Sov. Phys. Usp. 33, 403 (1990)].

[4] S. Murakami, N. Nagaosa, and S.-C. Zhang, Science 301, 1348 (2003); D. Culcer et al., Phys. Rev. Lett. 93, 046602 (2004); F. Zhou, Phys. Rev. B 70, 125321 (2004); A. Bérard and H. Mohrbach, Phys. Lett. A 352, 190 (2006); K.Yu. Bliokh and Yu.P. Bliokh, Ann. Phys. (N.Y.) 319, $13(2005)$.

[5] F.I. Fedorov, Dokl. Akad. Nauk SSSR 105, 465 (1955); C. Imbert, Phys. Rev. D 5, 787 (1972); M.A. Player, J. Phys. A: Math. Gen. 20, 3667 (1987); V.G. Fedoseyev, ibid. 21, 2045 (1988); W. Nasalski, J. Opt. Soc. Am. A 13, 172 (1996).

[6] V.S. Liberman and B.Ya. Zel'dovich, Phys. Rev. A 46, 5199 (1992).

[7] K.Yu. Bliokh and Yu.P. Bliokh Phys. Rev. E 70, 026605 (2004); Phys. Lett. A 333, 181 (2004); K.Yu. Bliokh and V.D. Freilikher, Phys. Rev. B 72, 035108 (2005).

[8] M. Onoda, S. Murakami, and N. Nagaosa, Phys. Rev. Lett. 93, 083901 (2004).

[9] C. Duval, Z. Horváth, and P.A. Horváthy, math-ph/0509031 cond-mat/0509636 (to appear in 
Phys. Rev D).

[10] K.Yu. Bliokh and Yu.P. Bliokh, Phys. Rev. Lett. 96, 073903 (2006).

[11] V.G. Fedoseyev, Opt. Comm. 193, 9 (2001); R. Dasgupta and P.K. Gupta, ibid. 257, 91 (2006).

[12] The field structure obeys the parallel transport law in diffractionless approximation. Diffraction deformations of the beam envelope, in general case, do not follow the parallel transport, P. Berczynski et al., J. Opt. Soc. Am. A 23, 1442 (2006). Screw form of the astigmatic beams, J.A. Arnaud and H. Kogelnik, Appl. Opt. 8, 1687 (1969), can also be attributed to the diffraction phenomena.

[13] A.W. Snyder and J.D. Love, Optical Waveguide Theory (Methuen, London, 1984).
[14] A.V. Dooghin et al., Phys. Rev. A 45, 8204 (1992).

[15] Unlike the small (proportional to the wavelength) effect of the rotation of the speckle-pattern in a direct fiber depending on the polarization [6, 14], (which can be attributed to weak spin-orbit interaction between SAM and IOAM), the proposed effect is of the order of unity and depends only on the torsion of fiber.

[16] B.A. Bernevig, T.L. Hughes, and S.-C. Zhang, Phys. Rev. Lett. 95, 066601 (2005).

[17] P. Ao and D.J. Thouless, ibid. 70, 2158 (1993).

[18] N.N. Punko and V.V. Filippov, Pis'ma v ZhETF 39, 18 (1984) [JETP Letters 39, 20 (1984)]. 